\title{
Some disassembly required: role of DNA translocases in the disruption of recombination intermediates and dead-end complexes
}

\author{
Lorraine S. Symington ${ }^{1,3}$ and Wolf-Dietrich Heyer ${ }^{2,4}$ \\ ${ }^{1}$ Department of Microbiology, Columbia University Medical Center, New York, New York 10032, USA; \\ ${ }^{2}$ Sections of Microbiology and of Molecular and Cellular Biology, Center for Genetics and Development, \\ University of California at Davis, Davis, California 95616, USA
}

Homologous recombination (HR) plays essential roles in the maintenance of genome integrity in prokaryotic and eukaryotic organisms. The primary function of HR in mitotic cells is to repair double-strand breaks (DSBs) or single-strand gaps that form as a result of replication fork collapse from processing of spontaneous damage and from exposure to DNA damaging agents. During meiosis, HR is essential to establish a physical connection between homologous chromosomes to ensure their correct disjunction at the first meiotic division. In meiosis, the high levels of recombination result from the programmed introduction of DSBs throughout the genome by the Spo11 transesterase. After breaks form in meiotic or mitotic cells, the ends are processed to yield 3' singlestrand DNA (ssDNA) tails that are active in strand invasion with a homologous duplex (Fig. 1). Following DNA strand invasion, the invading 3 ' end is extended by DNA synthesis. The displaced loop of the donor duplex can pair with the other side of the break (DSBR [DSB repair] model) and serve as a template for repair synthesis from the other 3' end. Gap filling and ligation results in the formation of a double Holliday junction (dHJ) intermediate that can be resolved to generate crossover or noncrossover products. Alternatively, the invading strand that was extended by DNA synthesis is displaced and pairs with the other side of the break (SDSA [synthesis-dependent strand annealing] model).

Central to the process of HR is the pairing of DNA molecules and exchange of single strands to form heteroduplex DNA, a reaction catalyzed by members of the RecA/Rad51 family of proteins. Besides the ubiquitous Rad51, which is important for recombinational repair of DSBs and ssDNA gaps in mitotic and meiotic cells, eu-

Correspondence.

${ }^{3}$ E-MAIL 1ss5@columbia.edu; FAX (212) 305-1741.

${ }^{4}$ E-MAIL wdheyer@ucdavis.edu; FAX (530) 752-3011.

Article is online at http://www.genesdev.org/cgi/doi/10.1101/gad.1477106. karyotes commonly have in addition the highly related Dmcl that is expressed only during meiosis (Bishop et al. 1992; Shinohara et al. 1992). These two proteins have partially overlapping but also unique functions in meiotic recombination (Shinohara et al. 1997a). RecA and Rad51 are the most extensively studied members of the family of homologous pairing and strand exchange proteins, and the reaction catalyzed is described briefly below. In the first step, RecA (or Rad51) assembles on ssDNA generated at resected DSBs or stalled replication forks to form a helical nucleoprotein filament that is active in homologous pairing. Although RecA preferentially binds to ssDNA (Kowalczykowski 1991), both Rad51 and Dmc1 bind almost equally well to doublestranded DNA (dsDNA) in vitro, raising the question of how these proteins are targeted to ssDNA and not sequestered nonspecifically on chromatin (Shinohara et al. 1992; Hong et al. 2001). Nucleation of Rad51 on dsDNA is inhibitory to DNA strand exchange in vitro, suggesting that this process might be actively discouraged in vivo to allow filament formation exclusively on ssDNA generated at sites of damage (Sung and Robberson 1995; Solinger et al. 2002). In this issue, Holzen et al. (2006) provide direct evidence that the Rdh54/Tid1 DNA translocase, a member of the Swi2/Snf2-like family of motor proteins, promotes dissociation of Dmcl from nonrecombinogenic sites on chromatin. This intriguing observation suggests eukaryotes may have evolved distinct mechanisms to maintain a free pool of Dmc1 or Rad51 available for DNA binding at the right time and the right place.

Once assembled, the RecA or Rad51 nucleoprotein filament is capable of interacting with a second DNA molecule to initiate strand exchange (Kowalczykowski 1991). Synapsis involves the alignment of the ssDNA within the nucleoprotein filament with homologous sequences within dsDNA. The recognition of homology and strand exchange are thought to be part of the same 


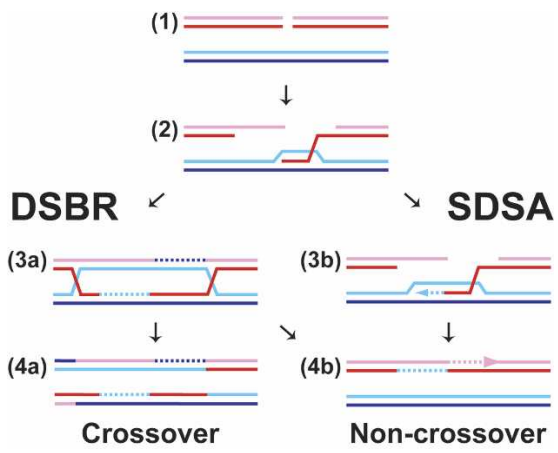

Figure 1. DSB repair by homologous recombination. After processing of the DSB, the homologous pairing and DNA-strandexchange proteins (Rad51, Dmc1) form a nucleoprotein filament with the help of mediator proteins $(\operatorname{Rad} 52$ and Rad55Rad57 for Rad51; Sae3-Mei5 and Hop2-Mnd1 for Dmc1 in S. cerevisiae) that invades the target sequence to form a D-loop (step 2) assisted by the Rad54 and Rdh54/Tid1 proteins (see Fig. 2). At the D-loop intermediate, homologous recombinations bifurcates into two subpathways. In DSBR, the second end is captured leading to the formation of a $\mathrm{dHJ}$ (step 3a) that is processed to crossover (step $4 \mathrm{a}$ ) and noncrossover (step $4 \mathrm{~b}$ ) products. In SDSA, the extended D-loop (step $3 \mathrm{~b}$ ) is dissolved, likely by a DNA helicase (see Fig. 2), and the newly synthesized strand reanneals with the second end of the DSB to restore a contiguous chromosome in noncrossover configuration (step 4b).

process and to occur by flipping of bases, predominately A:T base pairs, out of the helix in order to nucleate base pairing with the incoming ssDNA (Gupta et al. 1999). Once formed, heteroduplex DNA (hDNA) can be extended by branch migration. Finally, the DNA strand exchange protein is disassembled from the hDNA product, enabling access to the replication machinery to prime DNA synthesis from the invading 3 ' end and for processing of recombination intermediates. While this pathway has been considered a linear progression, accumulating evidence suggests several steps of the pathway are reversible to ensure assembly of recombination intermediates at the correct location and possibly to effect alternative modes of resolution. Several of the proteins that regulate recombination use the energy from ATP hydrolysis to translocate on DNA, thereby disrupting protein/DNA complexes and DNA intermediates; these include Rad54, Rdh54/Tid1, Srs2, and the RecQ-like DNA helicases Sgs1 and BLM.

\section{Rad54 dissociates Rad51-dsDNA filaments: disassembling unproductive complexes and moving the pathway along}

Much attention has been focused on the assembly of the Rad51 and Dmc1 filaments on ssDNA, the catalytically active complexes essential for homologous pairing and strand invasion. Proper assembly requires a host of mediator proteins in vivo and careful attention to the reaction conditions and stoichiometries in vitro (Krogh and Symington 2004). The disassembly of the filaments from the product heteroduplex DNA has been largely ignored experimentally and typically side-stepped in biochemical reactions by SDS-proteinase $\mathrm{K}$ treatment prior to product analysis. With RecA protein, ATP hydrolysis is required for product release and RecA turnover, leading to dynamic assembly/disassembly driven by nucleotide cofactor binding and hydrolysis cycles (Kowalczykowski 1991; Cox 2003). The eukaryotic RecA homolog Rad51 displays significantly ( 200-fold) less ATPase activity on dsDNA (Sung 1994) and is essentially stuck on the product DNA. How can eukaryotic cells compensate for this less dynamic behavior by their strand exchange protein? The answer lies with a very dynamic motor protein, Rad54, which engages in specific interaction with the Rad51 filaments on ssDNA and dsDNA.

$\operatorname{Rad} 54$ protein is a member of the Swi2/Snf2-like family of DNA-dependent ATPases comprising proteins that remodel a wide spectrum of protein-dsDNA complexes including nucleosomes, the TATA-binding protein (TBP)-dsDNA complex, and Rad51-dsDNA filaments (Tan et al. 2003; Heyer et al. 2006). Although this protein family is grouped within the super family 2 of DNA helicases, the members do not catalyze the melting of dsDNA typical of helicases, but rather they translocate on dsDNA, inducing topological changes and remodeling protein-dsDNA complexes. The Saccharomyces cerevisiae genome alone contains 17 Swi2/Snf2-like proteins, of which seven, including Rad54 and Rdh54/Tid1, function in various DNA repair/recombination pathways, presumably with distinct target complexes. Translocation on duplex by Rad54 was inferred from an assortment of bulk phase experiments (Tan et al. 2003; Heyer et al. 2006), and has now been directly visualized in single-molecule experiments, to move processively and surprisingly fast at $300 \mathrm{bp} / \mathrm{sec}$ (Amitani et al. 2006).

Rad54 is a core factor of HR machinery, and its biological function depends on its ATPase activity (Krogh and Symington 2004). Biochemical experiments demonstrated that Rad54 cooperates with Rad51 in complex ways during in vitro recombination reactions (Petukhova et al. 1998). The mechanisms involved are intricate and not entirely worked out (Tan et al. 2003; Heyer et al. 2006). First, Rad54 associates with the Rad51-ssDNA filaments, stabilizing them and delivering Rad54 to the pairing site (Fig. 2, step 2-3; Mazin et al. 2000, 2003). This function of Rad54 is independent of its ATPase activity, suggesting that other ATP-dependent functions are possibly more critical. However, in vivo ChIP experiments and cytological studies have demonstrated that Rad54 stabilizes Rad51-ssDNA filaments also in vivo (Wolner and Peterson 2005; Wesoly et al. 2006). Second, Rad54 stimulates Rad51-mediated DNA pairing and strand exchange in an ATP-dependent fashion (Fig. 2, step 3-4). Several potential mechanisms have been proposed (Van Komen et al. 2000; Ristic et al. 2001). The Rad54 motor may slide along the target duplex DNA to increase the efficiency of the homology search. Alternatively, the topological change induced by the motor may allow easier strand invasion by the Rad51 filament. Or, Rad54 may dissociate Rad51 from the duplex target DNA, which is known to inhibit pairing reactions (Sung 


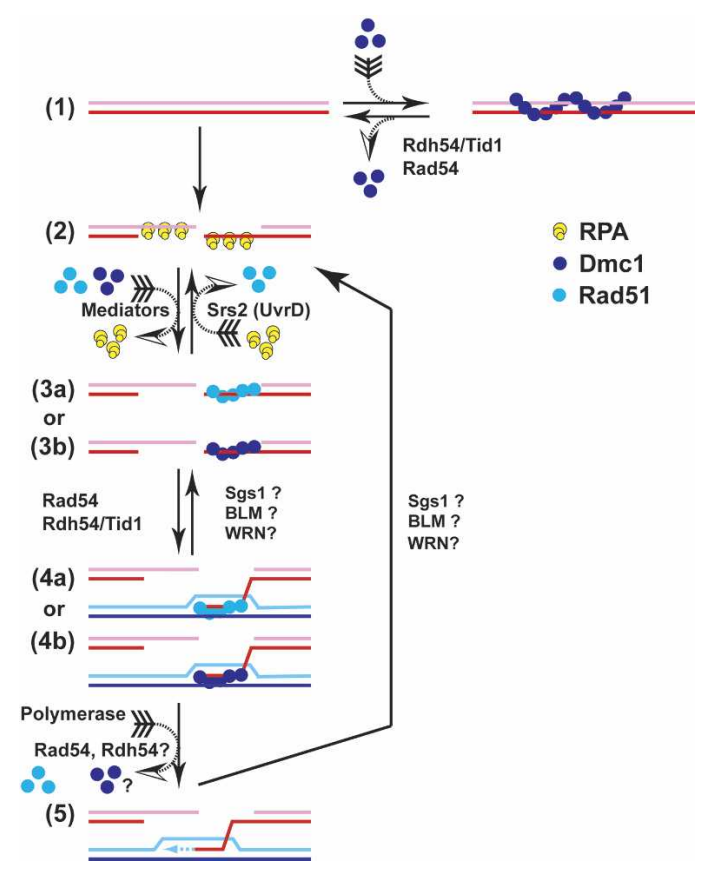

Figure 2. Dynamic balance in the assembly and disassembly of intermediates during homologous recombination. DNA-based motor proteins, helicases (Srs2, UvrD, Sgs1, BLM, WRN) and dsDNA translocases (Rad54, Rdh54/Tid1), have the ability to dissociate protein-DNA complexes and junction intermediates during homologous recombination. Rdh54/Tid1 (and Rad54?) dissociates Dmc1 from dsDNA (step 1) to feed a pool of free Dmc1 protein. Free Dmc1 and Rad51 protomers are utilized for assembly into ssDNA filaments during DSB-induced recombination (step 2-3). The DNA helicases Srs2 and UvrD catalyze the backward reaction of dissociating the Rad51-ssDNA and RecA-ssDNA filaments, respectively (step 3-2). It has not been tested whether Srs2 (or another helicase) can dissociate Dmc1ssDNA filaments. Rad54 and Rdh54/Tid1 facilitate D-loop formation by Rad51 (step 3-4) and can also dissociate the Rad51dsDNA filament, which is consistent with a function after DNA strand invasion (step 4-5). A similar function is envisioned by Holzen et al. (2006) for Rdh54/Tid1 dissociating the Dmc1-dsDNA product complex. Sgs1, BLM, and WRN helicases are candidate proteins to dissociate nascent D-loops (step 4-3) or extended D-loops (step 5 to steps 3 or 2) in a back reaction after initial synapsis.

and Robberson 1995; Solinger et al. 2002), as proposed by Holzen et al. (2006) to account for the function of Rdh54/ Tid1 in dissociating dead-end Dmc1-dsDNA complexes. The ability of Rad54 to remodel chromatin in vitro provides an additional potential mechanism for Rad54 function in vivo (Alexeev et al. 2003; Alexiadis and Kadonaga 2003; Jaskelioff et al. 2003). Third, Rad54's ability to dissociate Rad51 from dsDNA may lead to turnover of Rad51 from the product heteroduplex DNA (Fig. 2, step 4-5; Solinger et al. 2002). This function after DNA strand exchange may allow DNA polymerase to access the invading 3 '-OH end analogous to the ATP hydrolysis-dependent turnover by RecA that is required to allow Pol III holoenzyme access to the invading strand (Xu and Marians 2002). In addition, Rad54 has been found in vitro to branch migrate three- and four-stranded junctions (Solinger et al. 2001; Bugreev et al. 2006). These last postsynaptic functions of Rad54 are consistent with the genetic placement of Rad54 downstream from Rad51 as well as cytological studies that identified a defect in rad54 mutants in the turnover of Rad51 foci during recombination (Shinohara et al. 2000; Tan et al. 2003; Krogh and Symington 2004; Miyazaki et al. 2004; Heyer et al. 2006; Wesoly et al. 2006).

The cytological and physical studies pinpointed the rad54 defect to a step after Rad51 association with the broken DNA and association of the presynaptic filament with its target DNA, but before DNA synthesis from the strand invasion product (Sugawara et al. 2003; Wolner and Peterson 2005). However, these experiments failed to unequivocally distinguish between roles of Rad54 in synapsis or post-synapsis. It is unclear whether the Rad51 foci accumulating in rad54 cells represent presynaptic filaments, joint molecules, or both. Likewise, it is unclear whether in vivo Rad51-mediated pairing intermediates (D-loops) or their extension by DNA polymerase requires Rad54. Such intermediates have not been identified yet during DSB repair in vegetative cells. Although single-end invasion intermediates have been documented during meiotic recombination (Hunter and Kleckner 2001), the effect of rad54 and rdh54/tid1 single and double mutants on the formation of single end invasion intermediates is unknown.

With these caveats in mind, the biochemical and biophysical analyses of Rad54 protein and its interaction with Rad51-dsDNA filaments argue strongly for a biologically significant function of the Rad54 motor in dissociating Rad51-dsDNA filaments. The activity of Rad54 is stimulated by Rad51 DNA filaments (Mazin et al. 2000; Van Komen et al. 2000); in particular, its ATPase activity is specifically enhanced sixfold by partial Rad51-dsDNA filaments (Kiianitsa et al. 2002). Such filaments provide protein-free DNA for Rad54 translocation, and electron microscopy directly visualized Rad54 particles at the terminus of Rad51-dsDNA filaments (Kiianitsa et al. 2006). These findings are consistent with a model of Rad54 oligomers translocating along dsDNA (Amitani et al. 2006) and specifically targeting the Rad51 filament dissociating protomers from the terminus.

\section{Dissociation of Dmc1-dsDNA filaments by Rdh54/ Tid1: freeing up the pool of Dmc1 and release of Dmc1 from recombination intermediates?}

Rdh54/Tid1 is a structural and functional homolog of the Rad54 protein (Klein 1997; Shinohara et al. 1997b). Like Rad54, Rdh54/Tid1 is a robust dsDNA-activated ATPase that translocates on dsDNA generating unconstrained negative and positive supercoils, as well as disrupting Rad51-dsDNA filaments (Petukhova et al. 2000; Chi et al. 2006). Rdh54/Tid1 interacts directly with Rad51 and stimulates the pairing activity of Rad51 in vitro (Dresser et al. 1997; Petukhova et al. 2000). However, Rdh54/Tid1 is unable to fully substitute for Rad54 in vivo because the rad54 single mutant shows extreme 
sensitivity to DNA damaging agents, whereas the rdh54/ tid1 single mutant is only mildly sensitive (Krogh and Symington 2004). rad54 rdh54/tid1 double mutants show even higher sensitivity to DNA damaging agents than the single mutants, indicating some functional overlap between the proteins. Interestingly, the double mutant exhibits a more severe phenotype in diploids than in haploids, and the poor growth of diploid mitotic cells is suppressed by deletion of RAD51, indicating that it is due to the accumulation of toxic recombination intermediates (Klein 1997). The precise nature of these recombination intermediates is currently unknown. During meiosis Rdh54/Tid1 is more important than Rad54 for full levels of recombination between chromosome homologs (Shinohara et al. 1997b; Arbel et al. 1999). Rdh54/Tid1 also interacts with Dmc1 (Dresser et al. 1997), but no stimulation of Dmc1 DNA strand exchange by Rdh54/Tid1 has yet been reported.

Immunostaining of spread meiotic nuclei has revealed the presence of discrete subnuclear assemblies of Rad51 and Dmc1, referred to as foci (Bishop 1994). Rad51 and Dmc1 foci show frequent colocalization and foci formation is dependent on meiosis-specific DSBs (SPO11-dependent). Dmc1 foci formation is perturbed in $\mathrm{rdh} 54 /$ tid1 mutants with foci formation occurring earlier than in wild-type cells and with greatly reduced colocalization between Dmc1 and Rad51 (Shinohara et al. 2000). Dmc1 and Rad51 colocalization is further reduced in the rad54 rdh54/tid1 double mutant, indicating that Rad54 is able to partially substitute for Rdh54/Tid1 in the coordinated assembly of Rad51 and Dmcl complexes. Holzen et al. (2006) provide compelling evidence that the failure of Rad51 and Dmc1 to colocalize in the rad54 rdh54/tid1 double mutant is due to accumulation of Dmcl in static or dead-end complexes at non-DSB sites. Importantly, they show that Dmcl forms abundant bright foci in the rdh54/tid1 spo11 mutant, in which meiotic DSBs are not generated (Fig. 2). Although Dmc1 is still recruited to meiotic DSBs in the SPO11 rdh54/ tid1 strain, it shows higher association with non-DSB sites than seen in the SPO11 RDH54/TID1 strain (Fukuda and Ohya 2006; Holzen et al. 2006). In the absence of both Rad54 and Rdh54/Tid1, Dmc1 is no longer enriched at DSBs and still shows high nonspecific association with other sequences. The long-lived Dmc1 and Rad51 foci observed in rad54 rdh54/tid1 mutants could be due to sequestration of the RecA homologs on chromatin before DSB formation, accumulation of presynaptic complexes, failed turnover from hDNA at the end of recombination, or a combination of these defects. The study by Holzen et al. (2006) is the first to show that dissociation of Dmcl from chromatin is an important early step in recombination, presumably to maintain a free pool of Dmcl ready to be recruited to ssDNA at resected DSBs.

These observations raise the interesting question of whether Rad54 and/or Rdh54/Tid1 might play a similar role in displacing Rad51 from dsDNA to facilitate presynaptic filament formation. Overexpression of Rad54 suppresses the radiation sensitivity conferred by the
rad51-K191R mutation, which encodes a protein defective for ATP hydrolysis (Morgan et al. 2002). One explanation for this observation is that in the absence of ATP hydrolysis Rad51 is more dependent on Rad54 for protein turnover from chromatin to increase the pool of free Rad51. Alternatively, increased levels of Rad54 might facilitate pairing efficiency of a suboptimal presynaptic filament or enable turnover from the hDNA intermediate. The radiation sensitivity of the rad51-K191R strain is also suppressed by overexpression of the mutant Rad51 protein consistent with the view that the pool of protomers is limiting.

\section{Srs2/UvrD dissociate Rad51/RecA-ssDNA filaments: reversing an early step in the pathway}

The Rad54 and Rdh54/Tid1 motor proteins dissociate dead-end complexes to feed the protomer pool of Dmc1 (and possibly Rad51) (Chi et al. 2006; Holzen et al. 2006), as well as to dissociate the Rad51 (and possibly Dmc1) product complex to keep the recombination pathway moving forward (Solinger et al. 2002; Holzen et al. 2006). However, Srs2 and its bacterial counterpart, the related UvrD helicase, dissociate the Rad51(RecA)-ssDNA filaments to prevent inappropriate initiation of $\mathrm{HR}$ on ssDNA.

Srs2 was identified as a suppressor of rad6, which channels lesions to $\mathrm{HR}$ in the absence of alternative post-replication pathways (translesion synthesis, template switching). Cells defective in Srs2 display a significant hyper-recombination (hyper-rec) phenotype (Aguilera and Klein 1988), and elegant genetic experiments suggested a specific role of Srs2 in counteracting recombination by acting on Rad51 protein (Aboussekhra et al. 1992). This model was beautifully corroborated by direct biochemical experiments, demonstrating that Srs2 dissociate Rad51 from ssDNA (Krejci et al. 2003; Veaute et al. 2003). Srs2 exhibits $3^{\prime}-5^{\prime}$ DNA helicase activity (Rong and Klein 1993), suggesting that the protein translocates from the $3^{\prime}$ end to dissociate Rad51 from the end destined to invade the duplex DNA (Fig. 2). It is unclear whether its strand separation activity has any biological significance, although Srs2 is also known to function in other processes (Krogh and Symington 2004). The dissociation of Rad51-ssDNA filaments not only provides a very satisfying and specific mechanism for the Srs 2 antirecombination activity, it also suggests that a Rad51 filament stands in a dynamic balance with its assembly regulated by mediator proteins and disassembly promoted by Srs2 (C.W. Fung and L.S. Symington, unpubl.). The closest bacterial homolog of Srs2, UvrD, displays a near identical biochemical behavior with RecA, which is consistent with its recombination phenotypes (Veaute et al. 2005). Regulation of Srs2 is achieved by its recruitment to stalled replication forks through an interaction with sumoylated PCNA, which favors shunting stalled forks to the translesion DNA synthesis and template switching pathways and disfavors HR (Papouli et al. 2005; Pfander et al. 2005).

Srs2 lacks an obvious homolog in metazoans, but an 
Srs2-related protein, Fbh1, is present in mammals and in Schizosaccharomyces pombe (Morishita et al. 2005; Osman et al. 2005). It is unknown whether Fbh1 can disrupt Rad51-ssDNA filaments, but the $S$. pombe fbh1 mutation does suppress the requirement for mediators of Rad51 filament assembly, similar to the phenotype of srs2 in $S$. cerevisiae. Furthermore, the fbh1 mutation confers a synthetic growth defect with srs2 or rqh1 (encodes a RecQ homolog) mutations in $S$. pombe, which can be suppressed by a rad51 mutation, indicating the accumulation of toxic recombination intermediates in the helicase double mutants (Morishita et al. 2005; Osman et al. 2005). It is also possible that RecQ-like helicases have substituted for Srs2 function in metazoans, because Sgs1 overexpression can rescue an srs2 defect (Mankouri et al. 2002)

While Srs2 as an anti-recombination factor is quite well understood, Srs2 was also found to be required for efficient DSB repair by the SDSA pathway (Fig. 1; Aylon et al. 2003; Ira et al. 2003). This observation led to the proposal that Srs2 not only dissociates Rad51 from ssDNA but can also dissociate the D-loop to allow reannealing with the second end (Ira et al. 2003). However, Srs2 was unable to dissociate Rad51-made DNA joint molecules in a reconstituted system (Veaute et al. 2003). This suggests that Srs 2 might require other cofactors or conditions for such an activity. A prorecombinogenic role for Srs2 is also suggested by the observation of shorter gene conversion tracts in srs 2 mutants contributing to the hyper-rec phenotype for heteroallelic recombination. The hyper gene conversion phenotype is not seen for srs2 alleles that lack the PCNA interaction domain, suggesting a function of Srs2 distinct from the anti-recombinogenic role at stalled replication forks (Aguilera and Klein 1988; Pfander et al. 2005).

\section{Dissociation of D-loops by Sgs1/BLM helicases: dynamic invasion-dissociation-reinvasion cycles in recombination?}

While genetic evidence suggests that Srs2 might play a role in dissociating the extended D-loop to shunt this intermediate to the SDSA pathway (Fig. 1; Aylon et al. 2003; Ira et al. 2003), the biochemical analysis has not yet been able to recapitulate such a reaction. However, genetic and biochemical evidence supports such a role for Sgs1 and human BLM protein, two RecQ-like DNA helicases with complex and incompletely understood roles in HR.

Mutations in SGS1 confer puzzling recombination phenotypes. The mutants are hyper-rec for spontaneous recombination but hypo-rec for induced recombination (Gangloff et al. 2000; Onoda et al. 2001). Mutants defective for the BLM helicase display highly elevated sister chromatid exchange, which appears to be a direct consequence of the inability of BLM-deficient cells to dissolve dHJ into noncrossover products (Wu and Hickson 2003). Genetic data in yeast have been interpreted as Sgs1 playing a similar role also in S. cerevisiae (Ira et al. 2003; Rockmill et al. 2003), but these data are also consistent with other interpretations; for example, the dissolution of a D-loop intermediate by Sgs1. The dazzling complexity of the sgs1 (BLM) phenotypes may be related to the biochemical observation that these proteins can function either alone or with protein partners. The human BLM helicase has been shown to dissociate D-loops by itself (van Brabant et al. 2000). However, the dissolution of $\mathrm{dHJ}$ s requires both BLM and topoisomerase III $\alpha$, and is stimulated by BLAP75/Rmil (Wu et al. 2006). Genetic data in budding yeast also support the idea that Sgs1 functions alone and in combination with topoisomerase III (Krogh and Symington 2004). A possible role for BLM helicase in dissolving D-loops was proposed on the basis of genetic data in Drosophila (McVey et al. 2004a,b) suggesting a model of multiple invasion/dissolution cycles during SDSA. WRN, another member of the RecQ family of helicases, also promotes dissociation of D-loops in vitro, and this could be relevant to its anti-recombination function (Prince et al. 2001; Orren et al. 2002).

These findings suggest that DNA strand invasion intermediates (Fig. 2, steps 4, 5) are possibly in a dynamic equilibrium between their formation and dissolution. The ability to reverse an extended D-loop is an inherent feature of the SDSA pathway (Fig. 1) but may also be important to reverse homeologous interactions (Myung et al. 2001). The hypo-rec phenotype of yeast sgs1 mutants could reflect that this dynamic equilibrium is required under conditions where recombination has to deal with multiple lesions and limited time.

\section{Dynamic instability of recombination intermediates and DNA complexes of recombination proteins}

The recombination reaction catalyzed by the eukaryotic RecA homologs, Rad51 and Dmc1, is in a dynamic balance with regulation of forward and backward reactions by the energy-dependent assembly/disassembly of helical filaments and by translocating motor proteins. The initial assembly of the Rad51 or Dmc1-ssDNA filament is driven by ATP-dependent binding by Rad51 or Dmc1 with the aid of mediator proteins, and the disassembly of the Rad51 or Dmc1-dsDNA product complex is achieved by ATP hydrolysis and the extrinsic turnover factors, Rad54 and Rdh54/Tid1. This active assembly/ disassembly cycle of a helical filament driven by nucleotide cofactor binding/hydrolysis and extrinsic protein factors is reminiscent of the dynamic assembly and disassembly of actin filaments (Pollard and Borisy 2003). Such dynamic instability allows reorganization of the Rad51-Dmc1 filaments on ssDNA, which has been postulated to be required for the bypass of heterology by the related RecA protein (Kowalczykowski 1991). Moreover, as shown by Holzen et al. (2006), this dynamic instability allows disassembly of dead-end complexes to maintain a free pool of Dmcl protomers for recruitment to resected DSBs. Lastly, turnover of protein-DNA product complexes is likely required for progression through the recombination pathway (Fig. 2).

We propose dynamic instability to be an important and inherent feature of the entire recombination ma- 
chinery and not just limited to assembly and disassembly of RecA-like proteins (Fig. 2). HR is comprised of various subpathways, including DSBR and SDSA (see Fig. 1), as well as Break-Induced Replication and SingleStrand Annealing. SDSA requires the reversal of the extended D-loop (Fig. 1). We suggest that a number of DNA intermediates in the recombination pathways are in a dynamic balance between their assembly and disassembly, as proposed by Houston and Broach (2006) for synapsed intermediates. The presynaptic Rad51 (and likely Dmc1) filament appears to be in a balance between assembly favored by the mediators and disassembly catalyzed by Srs2 in budding yeast. The initial strand invasion product may be in a balance between its formation by the Rad51 or Dmc1 presynaptic filament and its dissolution by the BLM/Sgs1 DNA helicases. The D-loop intermediate may also exist in a balance between the extension of the invading strand by DNA polymerases aided by Rad54 or Rdh54 and D-loop disassembly mediated by BLM/Sgs1. Such dynamic balances avoid accumulation of long-lived intermediates that may pose obstacles to further steps in the pathway and allows the reversion of recombination intermediates or dissociation of dead-end complexes.

\section{Acknowledgments}

We thank W.K. Holloman for helpful comments on the manuscript and T. Doty for her proofreading.

\section{References}

Aboussekhra, A., Chanet, R., Adjiri, A., and Fabre, F. 1992. Semi-dominant suppressors of Srs2 helicase mutations of Saccharomyces cerevisiae map in the RAD51 gene, whose sequence predicts a protein with similarities to procaryotic RecA protein. Mol. Cell. Biol. 12: 3224-3234.

Aguilera, A. and Klein, H.L. 1988. Genetic control of intrachromosomal recombination in Saccharomyces cerevisiae. I. Isolation and genetic characterization of hyper-recombination mutations. Genetics 119: 779-790.

Alexeev, A., Mazin, A., and Kowalczykowski, S.C. 2003. Rad54 protein possesses chromatin-remodeling activity stimulated by a Rad51-ssDNA nucleoprotein filament. Nat. Struct. Biol. 10: 182-186.

Alexiadis, V. and Kadonaga, J.T. 2003. Strand pairing by Rad54 and Rad51 is enhanced by chromatin. Genes \& Dev. 16: $2767-2771$

Amitani, I., Baskin, R.J., and Kowalczykowski, S.C. 2006. Visualization of Rad54, a chromatin remodeling protein, translocating on single DNA molecules. Mol. Cell 23: 143-148.

Arbel, A., Zenvirth, D., and Simchen, G. 1999. Sister chromatidbased DNA repair is mediated by $R A D 54$, not by $D M C 1$ or TID1. EMBO J. 18: 2648-2658.

Aylon, Y., Liefshitz, B., Bitan-Banin, G., and Kupiec, M. 2003. Molecular dissection of mitotic recombination in the yeast Saccharomyces cerevisiae. Mol. Cell. Biol. 23: 1403-1417.

Bishop, D.K. 1994. RecA homologs Dmc1 and Rad51 interact to form multiple nuclear complexes prior to meiotic chromosome synapsis. Cell 79: 1081-1092.

Bishop, D.K., Park, D., Xu, L., and Kleckner, N. 1992. DMC1: A meiosis-specific yeast homolog of E. coli recA required for recombination, synaptonemal complex formation, and cell cycle progression. Cell 69: 439-456.

Bugreev, D.V., Mazina, O.M., and Mazin, A.V. 2006. Rad54 protein promotes branch migration of Holliday junctions. $\mathrm{Na}$ ture 442: 590-593.

Chi, P., Kwon, Y., Seong, C., Epshtein, A., Lam, I., Sung, P., and Klein, H.L. 2006. Yeast recombination factor Rdh54 functionally interacts with the Rad51 recombinase and catalyzes Rad51 removal from DNA. J. Biol. Chem. (in press).

Cox, M.M. 2003. The bacterial RecA protein as a motor protein. Annu. Rev. Microbiol. 57: 551-577.

Dresser, M.E., Ewing, D.J., Conrad, M.N., Dominguez, A.M., Barstead, R., Jiang, H., and Kodadek, T. 1997. DMC1 functions in a Saccharomyces cerevisiae meiotic pathway that is largely independent of the RAD51 pathway. Genetics 147: 533-544.

Fukuda, T. and Ohya, Y. 2006. Recruitment of RecA homologs Dmclp and Rad51p to the double-strand break repair site initiated by meiosis-specific endonuclease VDE (PI-SceI). Mol. Genet. Genomics 275: 204-214.

Gangloff, S., Soustelle, C., and Fabre, F. 2000. Homologous recombination is responsible for cell death in the absence of the Sgs1 and Srs2 helicases. Nat. Genet. 25: 192-194.

Gupta, R.C., Folta-Stogniew, E., O'Malley, S., Takahashi, M., and Radding, C.M. 1999. Rapid exchange of A:T base pairs is essential for recognition of DNA homology by human Rad51 recombination protein. Mol. Cell 4: 705-714.

Heyer, W.D., Li, X.R. Rolfsmeier, M. and Zhang, X.-P. 2006. Rad54: The Swiss Army knife of homologous recombination? Nucleic Acids Res. (in press).

Holzen, T.M., Shah, P.P., Olivares, H.A., and Bishop, D.K. 2006. Tid1/RDH54 promotes dissociation of Dmc1 from nonrecombinogenic sites on meitoic chromatin. Genes \& Dev. (this issue).

Hong, E.L., Shinohara, A., and Bishop, D.K. 2001. Saccharomyces cerevisiae Dmcl protein promotes renaturation of single-strand DNA (ssDNA) and assimilation of ssDNA into homologous super-coiled duplex DNA. J. Biol. Chem. 276: 41906-41912.

Houston, P.L. and Broach, J.R. 2006. The dynamics of homologous pairing during mating type interconversion in budding yeast. PLoS Genet. 2: e98.

Hunter, N. and Kleckner, N. 2001. The single-end invasion: An asymmetric intermediate at the double strand break to double Holliday junction transition of meiotic recombination. Cell 106: 59-70.

Ira, G., Malkova, A., Liberi, G., Foiani, M., and Haber, J.E. 2003. Srs2 and Sgs1-Top3 suppress crossovers during doublestrand break repair in yeast. Cell 115: 401-411.

Jaskelioff, M., Van Komen, S., Krebs, J.E., Sung, P., and Peterson, C.L. 2003. Rad54p is a chromatin remodeling enzyme required for heteroduplex joint formation with chromatin. $J$. Biol. Chem. 278: 9212-9218.

Kiianitsa, K., Solinger, J.A., and Heyer, W.D. 2002. Rad54 protein exerts diverse modes of ATPase activity on duplex DNA partially and fully covered with Rad51 protein. I. Biol. Chem. 277: 46205-46215.

- 2006. Terminal association of the Rad54 protein with the Rad51-dsDNA filament. Proc. Nat1. Acad. Sci. 103: 9767-9772.

Klein, H.L. 1997. RDH54, a RAD54 homologue in Saccharomyces cerevisiae, is required for mitotic diploid-specific recombination and repair and for meiosis. Genetics 147: 1533 1543.

Kowalczykowski, S.C. 1991. Biochemistry of genetic recombination: Energetics and mechanism of DNA strand exchange. 
Annu. Rev. Biophys. Biophys. Chem. 20: 539-575.

Krejci, L., Van Komen, S., Li, Y., Villemain, J., Reddy, M.S., Klein, H., Ellenberger, T., and Sung, P. 2003. DNA helicase Srs2 disrupts the Rad51 presynaptic filament. Nature 423: 305-309.

Krogh, B.O. and Symington, L.S. 2004. Recombination proteins in yeast. Annu. Rev. Genet. 38: 233-271.

Mankouri, H.W., Craig, T.J., and Morgan, A. 2002. SGS1 is a multicopy suppressor of srs2: Functional overlap between DNA helicases. Nucleic Acids Res. 30: 1103-1113.

Mazin, A.V., Bornarth, C.J., Solinger, J.A., Heyer, W.-D., and Kowalczykowski, S.C. 2000. Rad54 protein is targeted to pairing loci by the Rad51 nucleoprotein filament. Mol. Cell 6: $583-592$.

Mazin, A.V., Alexeev, A.A., and Kowalczykowski, S.C. 2003. A novel function of Rad54 protein-Stabilization of the Rad51 nucleoprotein filament. J. Biol. Chem. 278: 14029-14036.

McVey, M., Adams, M., Staeva-Vieira, E., and Sekelsky, J.J. 2004a. Evidence for multiple cycles of strand invasion during repair of double-strand gaps in Drosophila. Genetics 167: 699-705.

McVey, M., LaRocque, J.R., Adams, M.D., and Sekelsky, J.J. 2004b. Formation of deletions during double-strand break repair in Drosophila DmBlm mutants occurs after strand invasion. Proc. Natl. Acad. Sci. 101: 15694-15699.

Miyazaki, T., Bressan, D.A., Shinohara, M., Haber, J.E., and Shinohara, A. 2004. In vivo assembly and disassembly of Rad51 and Rad52 complexes during double-strand break repair. EMBO I. 23: 939-949.

Morgan, E.A., Shah, N., and Symington, L.S. 2002. The requirement for ATP hydrolysis by Saccharomyces cerevisiae Rad51 is bypassed by mating-type heterozygosity or RAD54 in high copy. Mol. Cell. Biol. 22: 6336-6343.

Morishita, T., Furukawa, F., Sakaguchi, C., Toda, T., Carr, A.M., Iwasaki, H., and Shinagawa, H. 2005. Role of the Schizosaccharomyces pombe F-Box DNA helicase in processing recombination intermediates. Mol. Cell. Biol. 25: 8074-8083.

Myung, K., Datta, A., Chen, C., and Kolodner, R.D. 2001. SGS1, the Saccharomyces cerevisiae homologue of BLM and WRN, suppresses genome instability and homeologous recombination. Nat. Genet. 27: 113-116.

Onoda, F., Seki, M., Miyajima, A., and Enomoto, T. 2001. Involvement of SGS1 in DNA damage-induced heteroallelic recombination that requires $R A D 52$ in Saccharomyces cerevisiae. Mol. Gen. Genet. 264: 702-708.

Orren, D.K., Theodore, S., and Machwe, A. 2002. The Werner syndrome helicase/exonuclease (WRN) disrupts and degrades D-loops in vitro. Biochemistry 41: 13483-13488.

Osman, F., Dixon, J., Barr, A.R., and Whitby, M.C. 2005. The F-Box DNA helicase Fbh1 prevents Rhp51-dependent recombination without mediator proteins. Mol. Cell. Biol. 25: 8084-8096.

Papouli, E., Chen, S.H., Davies, A.A., Huttner, D., Krejci, L., Sung, P., and Ulrich, H.D. 2005. Crosstalk between SUMO and ubiquitin on PCNA is mediated by recruitment of the helicase Srs2p. Mol. Cell 19: 123-133.

Petukhova, G., Stratton, S., and Sung, P. 1998. Catalysis of homologous DNA pairing by yeast Rad51 and Rad54 proteins. Nature 393: 91-94.

Petukhova, G., Sung, P., and Klein, H. 2000. Promotion of Rad51-dependent D-loop formation by yeast recombination factor Rdh54/Tid1. Genes \& Dev. 14: 2206-2215.

Pfander, B., Moldavan, G.L., Sacher, M., Hoege, C., and Jentsch, S. 2005. SUMO-modified PCNA recruits Srs2 to prevent recombination during S phase. Nature 436: 428-433.
Pollard, T.D. and Borisy, G.G. 2003. Cellular motility driven by assembly and disassembly of actin filaments. Cell 112: 453465.

Prince, P.R., Emond, M.J., and Monnat Jr., R.J. 2001. Loss of Werner syndrome protein function promotes aberrant mitotic recombination. Genes \& Dev. 15: 933-938.

Ristic, D., Wyman, C., Paulusma, C., and Kanaar, R. 2001. The architecture of the human Rad54-DNA complex provides evidence for protein translocation along DNA. Proc. Natl. Acad. Sci. 98: 8454-8460.

Rockmill, B., Fung, J.C., Branda, S.S., and Roeder, G.S. 2003. The Sgs1 helicase regulates chromosome synapsis and meiotic crossing over. Curr. Biol. 13: 1954-1962.

Rong, L. and Klein, H.L. 1993. Purification and characterization of the SRS2 DNA helicase of the yeast Saccharomyces cerevisiae. J. Biol. Chem. 268: 1252-1259.

Shinohara, A., Ogawa, H., and Ogawa, T. 1992. Rad51 protein involved in repair and recombination in $S$. cerevisiae is a RecA-like protein. Cell 69: 457-470.

Shinohara, A., Gasior, S., Ogawa, T., Kleckner, N., and Bishop, D.K. 1997a. Saccharomyces cerevisiae recA homologues RAD51 and DMC1 have both distinct and overlapping roles in meiotic recombination. Genes Cells 2: 615-629.

Shinohara, M., Shita-Yamaguchi, E., Buerstedde, J.M., Shinagawa, H., Ogawa, H., and Shinohara, A. 1997b. Characterization of the roles of the Saccharomyces cerevisiae RAD54 gene and a homologue of RAD54, RDH54/TID1, in mitosis and meiosis. Genetics 147: 1545-1556.

Shinohara, M., Gasior, S.L., Bishop, D.K., and Shinohara, A. 2000. Tid1/Rdh54 promotes colocalization of Rad51 and Dmc1 during meiotic recombination. Proc. Natl. Acad. Sci. 97: 10814-10819.

Solinger, J.A., Lutz, G., Sugiyama, T., Kowalczykowski, S.C., and Heyer, W.D. 2001. Rad54 protein stimulates heteroduplex DNA formation in the synaptic phase of DNA strand exchange via specific interactions with the presynaptic Rad51 nucleoprotein filament. J. Mol. Biol. 307: 1207-1221.

Solinger, J.A., Kiianitsa, K., and Heyer, W.-D. 2002. Rad54, a Swi2/Snf2-like recombinational repair protein, disassembles Rad51:dsDNA filaments. Mol. Cell 10: 1175-1188.

Sugawara, N., Wang, X., and Haber, J.E. 2003. In vivo roles of Rad52, Rad54, and Rad55 proteins in Rad51-mediated recombination. Mol. Cell 12: 209-219.

Sung, P. 1994. Catalysis of ATP-dependent homologous DNA pairing and strand exchange by yeast RAD51 protein. Science 265: 1241-1243.

Sung, P. and Robberson, D.L. 1995. DNA strand exchange mediated by a RAD51-ssDNA nucleoprotein filament with polarity opposite to that of RecA. Cell 82: 453-461.

Tan, T.L.R., Kanaar, R., and Wyman, C. 2003. Rad54, a Jack of all trades in homologous recombination. DNA Repair (Amst.) 2: 787-794.

van Brabant, A.J., Ye, T., Sanz, M., German, J.L., Ellis, N.A., and Holloman, W.K. 2000. Binding and melting of D-loops by the Bloom syndrome helicase. Biochemistry 39: 14617-14625.

Van Komen, S., Petukhova, G., Sigurdsson, S., Stratton, S., and Sung, P. 2000. Superhelicity-driven homologous DNA pairing by yeast recombination factors Rad51 and Rad54. Mol. Cell 6: 563-572.

Veaute, X., Jeusset, J., Soustelle, C., Kowalczykowski, S.C., Le Cam, E., and Fabre, F. 2003. The Srs2 helicase prevents recombination by disrupting Rad51 nucleoprotein filaments. Nature 423: 309-312.

Veaute, X., Delmas, P., Selva, M., Jeusset, J., Le Cam, E., Matic, I., Fabre, F., and Petit, M.A. 2005. UvrD helicase, unlike Rep helicase, dismantles RecA nucleoprotein filaments in Esch- 
erichia coli. EMBO J. 24: 180-189.

Wesoly, J., Agarwal, S., Sigurdsson, S., Bussen, W., Van Komen, S., Qin, J.A., van Steeg, H., van Benthem, J., Wassenaar, E., Baarends, W.M., et al. 2006. Differential contributions of mammalian Rad54 paralogs to recombination, DNA damage repair, and meiosis. Mol. Cell. Biol. 26: 976-989.

Wolner, B. and Peterson, C.L. 2005. ATP-dependent and ATPindependent roles for the Rad54 chromatin remodeling enzyme during recombinational repair of a DNA double strand break. J. Biol. Chem. 280: 10855-10860.

Wu, L.J. and Hickson, I.D. 2003. The Bloom's syndrome helicase suppresses crossing-over during homologous recombination. Nature 426: 870-874.

Wu, L., Bachrati, C.Z., Ou, J., Xu, C., Yin, J., Chang, M., Wang, W., Li, L., Brown, G.W., and Hickson, I.D. 2006. BLAP75/ RMI1 promotes the BLM-dependent dissolution of homologous recombination intermediates. Proc. Natl. Acad. Sci. 103: 4068-4073.

Xu, L. and Marians, K.J. 2002. A dynamic RecA filament permits DNA polymerase-catalyzed extension of the invading strand in recombination intermediates. J. Biol. Chem. 277: 14321-14328. 


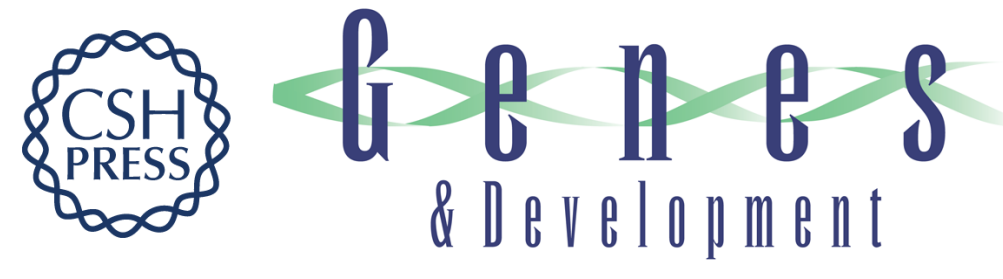

\section{Some disassembly required: role of DNA translocases in the disruption of recombination intermediates and dead-end complexes}

Lorraine S. Symington and Wolf-Dietrich Heyer

Genes Dev. 2006, 20:

Access the most recent version at doi:10.1101/gad.1477106

\section{Related Content Tid1/Rdh54 promotes dissociation of Dmc1 from nonrecombinogenic sites on meiotic chromatin \\ Teresa M. Holzen, Parisha P. Shah, Heidi A. Olivares, et al. \\ Genes Dev. September , 2006 20: 2593-2604}

References This article cites 65 articles, 27 of which can be accessed free at:

http://genesdev.cshlp.org/content/20/18/2479.full.html\#ref-list-1

Articles cited in:

http://genesdev.cshlp.org/content/20/18/2479.full.html\#related-urls

\section{License}

Email Alerting

Service

Receive free email alerts when new articles cite this article - sign up in the box at the top right corner of the article or click here.

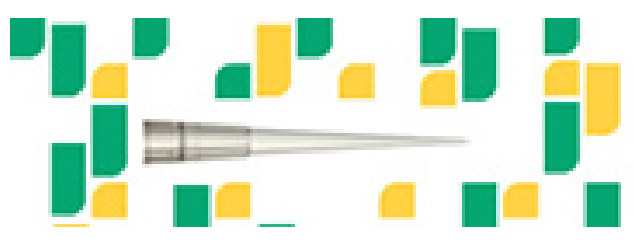

Focused on your science. 